\title{
Collective motions of a quantum gas confined in a harmonic trap
}

\author{
Dae-Yup Song \\ Department of Physics, Sunchon National University, Suncheon 540-742, Korea
}

(Dated: September 25, 2018)

\begin{abstract}
Single-component quantum gas confined in a harmonic potential, but otherwise isolated, is considered. From the invariance of the system of the gas under a displacement-type transformation, it is shown that the center of mass oscillates along a classical trajectory of a harmonic oscillator. It is also shown that this harmonic motion of the center has, in fact, been implied by Kohn's theorem. If there is no interaction between the atoms of the gas, the system in a time-independent isotropic potential of frequency $\nu_{c}$ is invariant under a squeeze-type unitary transformation, which gives collective radial breathing motion of frequency $2 \nu_{c}$ to the gas. The amplitudes of the oscillating and breathing motions from the exact invariances could be arbitrarily large. For a Fermi system, appearance of $2 \nu_{c}$ mode of the large breathing motion indicates that there is no interaction between the atoms, except for a possible long-range interaction through the inverse-square-type potential.
\end{abstract}

PACS numbers: 03.75.Ss, 11.30.Na, 03.75.Kk

Experiments at ultracold temperature have stimulated theoretical efforts to explore the properties of quantum many-body systems in harmonic potentials. As the interaction between the atoms of the quantum gas is controllable [1], the density profile of $N$-body noninteracting fermions has been investigated from the eigenstates of a harmonic oscillator [2]. For a onedimensional harmonic oscillator, it is known that the time-dependent Schrödinger equation is invariant under the displacement-type transformation [3], and squeezetype transformation (up to a rescaling of time) [4]. The invariance under the displacement-type transformation (DTT) gives wave functions whose probability distributions move along classical solutions. The invariance under the squeeze-type transformation (STT) gives breathing motions to the distributions. For a time-independent potential of frequency $\nu_{c}$, it is numerically shown that the breathing motion has frequency $2 \nu_{c}[5]$.

Harmonic motions of the centers of masses of the quantum gases have been clearly noticed in experiments and used to accurately measure the potentials (see, e.g., [ 6 ) ). Theoretically, dipole mode in the motion of the center has been found through various approximations, mainly based on the Gross-Pitaevskii (GP) mean-field formalism [5, 7]. In addition, for the time-independent potential, Kohn's theorem says the existence of exact excited states with excitation energies $2 \pi l \hbar \nu_{c}(l=1,2, \cdots)$ and further "harmonic-potential theorem" has been established [8]. To my knowledge, however, the harmonic motion of the center has rather been understood from the insight that "collisions between atoms cannot alter center-of-mass momentum" [6] in the context of ultracold temperature physics. The implication of the breathing motion of the harmonic oscillator to the Bose-Einstein condensates has also long been studied in literature. In particular, it has been suggested that the GP equation of a time-dependent harmonic trap may be transformed to the equation of a time-independent potential through the unitary transformation of a harmonic oscillator [9], and a scheme to understand the $2 \nu_{c}$ mode is proposed [10].
Recently, a $2 \nu_{c}$ mode has been found in an experiment [11.

In this paper, single-component quantum gas confined in a time-dependent harmonic potential, but otherwise isolated, is considered, with an assumption that positiondependent part of the interaction between the atoms of the gas is written in terms of the differences of positions of two atoms. From the exact invariance of the gas system under a DTT, it is shown that the center of mass oscillates along a classical trajectory of a harmonic oscillator as it has been known for the time-independent potential through a different formalism [8]. It is also shown that this harmonic motion of the center has, in fact, been implied by Kohn's theorem. If the interaction between the atoms is absent, the system in a time-independent isotropic $\nu_{c}$ potential is invariant under a STT up to a rescaling of time, which predicts the collective radial breathing motion of frequency $2 \nu_{c}$ of the gas. The amplitudes of the oscillating and breathing modes from the exact invariances could be arbitrarily large, as they are determined purely by the classical solutions. In this respect, the exact modes may be different from other modes found in the linear approximations [7]. For a Fermi system, appearance of $2 \nu_{c}$ mode of the large breathing motion signals that the gas is in the region of no interaction between the atoms, except for a possible long-range interaction through the inverse-square-type potential. The collective harmonic motions of the fermions will show that the complete set of the numbered coherent states 12, 13, 14 of a harmonic oscillator can be used in stacking the fermions, with equal validity, as the eigenstates are used. For simplicity, the nonlinear Schrödinger (generalized GP) equation will be considered first, and the formalism will be extended to the many-body systems.

A $D$-dimensional nonlinear Schrödinger equation (NLSE) with a time-dependent harmonic potential is given by

$$
O(t, w(t)) \Psi(\vec{r}, t)+g|\Psi(\vec{r}, t)|^{2 n} \Psi(\vec{r}, t)=0,
$$


with

$$
O(t, w(t))=-i \hbar \frac{\partial}{\partial t}-\frac{\hbar^{2} \vec{\nabla}^{2}}{2 m}+\frac{m}{2} \sum_{i=1}^{D} w_{i}^{2}(t) x_{i}^{2},
$$

where $\vec{\nabla}=\left(\frac{\partial}{\partial x_{1}}, \frac{\partial}{\partial x_{2}}, \cdots \frac{\partial}{\partial x_{D}}\right), m$ is the mass of an atom, and $g, n$ are constants. When $f_{i}$ satisfies the Hill's equation, the classical equation of motion of a harmonic oscillator,

$$
\ddot{f}_{i}+w_{i}^{2}(t) f_{i}=0
$$

with the overdot denoting differentiation with respect to $t$, the displacement-type unitary operator for the $D$ dimensional harmonic oscillator can be given as

$$
U_{f}(\vec{r}, t)=\prod_{i=1}^{D}\left(e^{\left[\frac{i}{\hbar}\left(\delta_{i}+m \dot{f}_{i} x_{i}\right)\right]} \exp \left[-f_{i} \frac{\partial}{\partial x_{i}}\right]\right),
$$

where $\delta_{i}$ is defined through the relation

$$
\dot{\delta}_{i}=\frac{1}{2} m\left(w_{i}^{2}(t) f_{i}^{2}-\dot{f}_{i}^{2}\right) .
$$

Making use of the operator, one can find that

$$
\begin{aligned}
\tilde{\Psi}(\vec{r}, t) & \equiv U_{f}(\vec{r}, t) \Psi(\vec{r}, t), \\
& =\left(\prod_{i=1}^{D} \exp \left[\frac{i}{\hbar}\left(\delta_{i}+m \dot{f}_{i} x_{i}\right)\right]\right) \Psi(\vec{r}-\vec{f}, t),
\end{aligned}
$$

with $\vec{f}=\left(f_{1}, f_{2}, \cdots, f_{D}\right)$. If the unitary operator $U_{f}(\vec{r}, t)$ is applied on (1), from the relation

$$
U_{f}(\vec{r}, t) O(t, w(t)) U_{f}^{\dagger}(\vec{r}, t)=O(t, w(t))
$$

and the fact that $U_{f}(\vec{r}, t)\left[|\Psi(\vec{r}, t)|^{2} \Psi(\vec{r}, t)\right]=$ $|\tilde{\Psi}(\vec{r}, t)|^{2} \tilde{\Psi}(\vec{r}, t)$, one can find that the transformed equation is the same one with the replacement of $\Psi(\vec{r}, t)$ by $\tilde{\Psi}(\vec{r}, t)$. If $\Psi(\vec{r}, t)$ is a solution of $(1), \tilde{\Psi}(\vec{r}, t)$ is also a solution of the same equation, and the number density $|\tilde{\Psi}(\vec{r}, t)|^{2}$ of the transformed solution has harmonic motions with respect to the original one, without changing the shape. If $w_{i}(t)$ is a constant $w_{c i}$ and the transformation is applied on a stationary solution of (1), then the number density of the transformed solution oscillates sinusoidally along the $i$-th direction. For $D=1$, the invariance has been known through a different formalism 15].

The invariance under the DTT can be easily extended for a $N$-body system, if the interaction $V(\vec{r}(1), \vec{r}(2), \cdots, \vec{r}(N))$ between the atoms satisfies

$$
\begin{aligned}
& V(\vec{r}(1)+\vec{c}, \vec{r}(2)+\vec{c}, \cdots, \vec{r}(N)+\vec{c}) \\
& =V(\vec{r}(1), \vec{r}(2), \cdots, \vec{r}(N)),
\end{aligned}
$$

where $\vec{r}(j)$ denotes the position of the $j$-th atom and $\vec{c}$ is a constant vector. By defining $U_{N}$ as

$$
U_{N}=\prod_{j=1}^{N} U_{f}(\vec{r}(j), t)
$$

one may find the relation

$$
U_{N} O_{N}(t, w(t)) U_{N}^{\dagger}=O_{N}(t, w(t)),
$$

where

$$
\begin{aligned}
& O_{N}(t, w(t)) \equiv-i \hbar \frac{\partial}{\partial t}+H_{N} \\
& =-i \hbar \frac{\partial}{\partial t}+V(\vec{r}(1), \vec{r}(2), \cdots, \vec{r}(N)) \\
& +\sum_{j=1}^{N}\left[-\frac{\hbar^{2} \vec{\nabla}^{2}(j)}{2 m}+\frac{m}{2} \sum_{i=1}^{D} w_{i}^{2}(t) x_{i}^{2}(j)\right] .
\end{aligned}
$$

For a given time, the operations of $U_{N}$ and $U_{f}(\vec{r}, t)$ amount to moving the space coordinate along the vector $\vec{f}$ to find new solutions, up to the multiplicative phase factors. The probability distribution of the transformed wave function of the $N$-body system will thus have the harmonic motion with respect to the distribution of the original wave function, as the number density of the transformed solution of the NLSE does. (10) is valid for both of the Fermi-Dirac and Bose-Einstein statistics, and is an exact explanation for the longstanding observation that the center of mass of the single-component gas confined in a harmonic potential moves harmonically, while the harmonic motion of the center has been known for the time-independent potential [8].

For the case of constant frequency $w_{i}(t)=w_{c i}(i=$ $1,2, \cdots, D)$, one may find that the harmonic motions of the centers of masses have, in fact, been implied by Kohn's theorem [8]. For this, by letting

$$
f_{i}=\sqrt{\frac{2 \hbar}{m w_{c i}}} z_{0 i} \cos \left(w_{c i} t+\varphi_{i}\right)
$$

with real constants $z_{0 i}, \varphi_{i}$, one can find the relations

$$
\begin{gathered}
\exp \left[\frac{i}{\hbar} \delta_{i}+\frac{i}{\hbar} m \dot{f}_{i} x_{i}(j)\right] \exp \left[-f_{i} \frac{\partial}{\partial x_{i}(j)}\right] \\
=e^{i \phi_{i}} \exp \left[\frac{i}{\hbar} m \dot{f}_{i} x_{i}(j)-f_{i} \frac{\partial}{\partial x_{i}(j)}\right] \\
=e^{i \phi_{i}} \exp \left[z_{i}(t) a_{i}^{\dagger}(j)-z_{i}^{*}(t) a_{i}(j)\right],
\end{gathered}
$$

where

$$
\begin{aligned}
& z_{i}(t)=z_{0 i} e^{-i\left(w_{c i} t+\varphi_{i}\right)}, \\
& a_{i}(j)=\frac{1}{\sqrt{2}}\left(\sqrt{\frac{m w_{c i}}{\hbar}} x_{i}(j)+\sqrt{\frac{\hbar}{m w_{c i}}} \frac{\partial}{\partial x_{i}(j)}\right),
\end{aligned}
$$

and $z_{i}^{*}(t)$ denotes complex conjugate of $z_{i}(t)$. In $(15,16)$, $\phi_{i}$ is a real constant coming from that $\delta_{i}$ is defined up to a constant, and from now on we will set $\phi_{i}=0(i=$ $1,2, \cdots, D)$. One can thus find that $U_{N}$ is written as

$$
\begin{aligned}
U_{N} & =\prod_{i=1}^{D} \exp \left(z_{i}(t) A_{i}^{\dagger}-z_{i}^{*}(t) A_{i}\right) \\
& =\prod_{i=1}^{D} e^{-N z_{0 i}^{2} / 2} \exp \left(z_{i}(t) A_{i}^{\dagger}\right) \exp \left(-z_{i}^{*}(t) A_{i}\right)
\end{aligned}
$$


where

$$
A_{i}=\sum_{j=1}^{N} a_{i}(j) .
$$

Indeed, in Ref. [16], it has been proven that

$$
\left[H_{N}, A_{i}^{\dagger}\right]=\hbar w_{c i} A_{i}^{\dagger} .
$$

If $\mid G>$ is the many-body ground state of $H_{N}$ with energy eigenvalue $E_{G}$, a wave function of the system is given as

$$
\begin{aligned}
& e^{-\frac{i}{\hbar} E_{G} t} U_{N} \mid G> \\
& =e^{-\frac{i}{\hbar} E_{G} t} \prod_{i=1}^{D} e^{-N z_{0 i}^{2} / 2}\left(\sum_{l=0}^{\infty} \frac{z_{i}^{l}}{l !}\left(A_{i}^{\dagger}\right)^{l} \mid G>\right),
\end{aligned}
$$

while, as suggested by Kohn's theorem [8], $\left(A_{i}^{\dagger}\right)^{l} \mid G>$ is an eigenstate of the $H_{N}$ with the eigenvalue $E_{G}+l \hbar w_{c i}$ [16].

From now on, isotropic potentials will only be considered, so that $w_{i}(t)=w(t)$ for all $i$. For the STT, the unitary operator

$$
\begin{aligned}
& U_{s} \\
& =\left(\frac{\Omega}{m w_{c} \eta^{2}(t)}\right)^{\frac{D}{4}} \exp \left[\frac{i}{2 \hbar} \frac{m \dot{\eta}(t)}{\eta(t)} \vec{r}^{2}\right] \\
& \quad \times \exp \left[-\frac{1}{2}\left(\ln \frac{m w_{c} \eta^{2}(t)}{\Omega}\right) \vec{r} \cdot \vec{\nabla}\right]
\end{aligned}
$$

is introduced, with positive constants $\Omega$ and $w_{c}\left(=2 \pi \nu_{c}\right)$. For the time being, it will be assumed that $\eta(t)$ is an arbitrary smooth positive function of $t$. If $U_{s}$ is applied on $\chi(\vec{r}, \tau(t))$, the transformed function will be

$$
\begin{aligned}
\Psi(\vec{r}, t)= & U_{s} \chi(\vec{r}, \tau(t)) \\
= & \left(\frac{\Omega}{m w_{c} \eta^{2}(t)}\right)^{\frac{D}{4}} \exp \left[\frac{i}{2 \hbar} \frac{m \dot{\eta}(t)}{\eta(t)} \vec{r}^{2}\right] \\
& \times \chi\left(\sqrt{\frac{\Omega}{m w_{c}}} \frac{\vec{r}}{\eta(t)}, \tau(t)\right) .
\end{aligned}
$$

A NLSE with a time-independent harmonic potential is given as

$$
O_{c}\left(\tau(t), w_{c}\right) \chi(\vec{r}, \tau(t))+g|\chi(\vec{r}, \tau(t))|^{2 n} \chi(\vec{r}, \tau(t))=0,
$$

where

$$
O_{c}\left(\tau(t), w_{c}\right)=-i \hbar \frac{\partial}{\partial \tau}-\frac{\hbar^{2} \vec{\nabla}^{2}}{2 m}+\frac{m w_{c}^{2}}{2} \vec{r}^{2} .
$$

From the fact

$$
\begin{aligned}
& U_{s}\left(|\chi(\vec{r}, \tau)|^{2 n} \chi(\vec{r}, \tau)\right) \\
& =\left(\frac{\Omega}{m w_{c} \eta^{2}(t)}\right)^{-\frac{n D}{2}}|\Psi(\vec{r}, t)|^{2 n} \Psi(\vec{r}, t),
\end{aligned}
$$

one may find that the NLSE of (23) can be transformed, through the STT, to that of (1) only when

$$
\frac{d t}{d \tau}=\frac{m w_{c} \eta^{2}}{\Omega} .
$$

Further, if $\eta(t)$ satisfies

$$
m \ddot{\eta}+m w^{2}(t) \eta-\frac{\Omega^{2}}{m \eta^{3}}=0,
$$

one can find the relation

$$
U_{s} O_{c}\left(\tau(t), w_{c}\right) U_{s}^{\dagger}=\left(\frac{d t}{d \tau}\right) O(t, w(t))
$$

which is well-known for the one-dimensional harmonic oscillators [4, 14]. When $\tau$ satisfies (26), by applying the operator $U_{s}$ on (23), one can find that the NLSE is transformed as

$$
\begin{aligned}
& U_{s}\left[O_{c}\left(\tau(t), w_{c}\right) \chi(\vec{r}, \tau(t))+g|\chi(\vec{r}, \tau(t))|^{2 n} \chi(\vec{r}, \tau(t))\right] \\
& =\frac{m w_{c} \eta^{2}(t)}{\Omega} \\
& \times\left[\begin{array}{l}
O(t, w(t)) \Psi(\vec{r}, t) \\
+g\left(\frac{\Omega}{m w_{c} \eta^{2}(t)}\right)^{1-\frac{n D}{2}}|\Psi(\vec{r}, t)|^{2 n} \Psi(\vec{r}, t)
\end{array}\right]=0 .
\end{aligned}
$$

Through the STT, (23) is thus transformed to (1) when

$$
n D=2 .
$$

As far as a NLSE is concerned, this condition is identical to that given in Ref. 10], found in a different context using a trial function. For $n=1,2$, the squeeze-type relation (29) has been suggested in Ref. [9].

In the harmonic oscillator, it is known that $\eta(t)$ and a constant $\Omega$ are written as (see, for example, Refs. [4, 13])

$$
\eta(t)=\sqrt{u^{2}(t)+v^{2}(t)}, \quad \Omega=m[\dot{v}(t) u(t)-\dot{u}(t) v(t)],
$$

with two linearly independent real solutions $u(t)$ and $v(t)$ of

$$
\ddot{x}_{c l}+w^{2}(t) x_{c l}=0 .
$$

From now on, we only consider $\tau(t)$ and $\eta(t)$ satisfying $(26,27)$. When $(30)$ is satisfied, the unitary relations can be used to find a solution $\Psi(\vec{r}, t)$ of (1) from a known solution $\chi(\vec{r}, \tau(t))$ of $(23)$, as

$$
\Psi(\vec{r}, t)=U_{f}(\vec{r}, t) U_{s} \chi(\vec{r}, \tau(t)) .
$$

If $e^{-i \mu \tau(t)} \chi_{0}(\vec{r})$ is a solution of (23) with a constant $\mu$, a solution of (1) is given as

$$
\begin{aligned}
& \Psi(\vec{r}, t) \\
& =\left(\frac{\Omega}{m w_{c} \eta^{2}(t)}\right)^{\frac{D}{4}}\left(\frac{u(t)-i v(t)}{\eta(t)}\right)^{\mu / \hbar} \\
& \quad \times\left(\prod_{i=1}^{D} \exp \left[\frac{i}{\hbar}\left(\delta_{i}+m \dot{f}_{i} x_{i}\right)\right]\right) \\
& \quad \times \exp \left[\frac{i m \dot{\eta}(t)}{2 \hbar \eta(t)}(\vec{r}-\vec{f})^{2}\right] \chi_{0}\left(\sqrt{\frac{\Omega}{m w_{c}}} \frac{\vec{r}-\vec{f}}{\eta(t)}\right) .
\end{aligned}
$$


For the case of $\vec{f}=0$, one finds that

$$
|\Psi(\vec{r}, t)|^{2}=\left(\sqrt{\frac{\Omega}{m w_{c}}} \frac{1}{\eta(t)}\right)^{D}\left|\chi_{0}\left(\sqrt{\frac{\Omega}{m w_{c}}} \frac{\vec{r}}{\eta(t)}\right)\right|^{2} .
$$

For a given time with $\vec{f}=0,(35)$ shows that the number density of $\Psi(\vec{r}, t)$ is given from that of $e^{-i \mu \tau(t)} \chi_{0}(r)$ by globally rescaling the space coordinate along the radial direction, with a multiplication factor needed to keep the total number of particles.

The squeeze-type unitary relation can be extended for the interacting $N$-body system, if interaction between the atoms is of homogeneous degree -2 , so that

$$
V(a \vec{r}(1), a \vec{r}(2), \cdots, a \vec{r}(N))=a^{-2} V(\vec{r}(1) \vec{r}(2), \cdots, \vec{r}(N))
$$

with a constant $a$. By defining

$$
\begin{aligned}
& O_{N, c}\left(\tau(t), w_{c}\right) \\
& =-i \hbar \frac{\partial}{\partial \tau}+V(\vec{r}(1), \vec{r}(2), \cdots, \vec{r}(N)) \\
& +\sum_{j=1}^{N}\left[-\frac{\hbar^{2} \vec{\nabla}^{2}(j)}{2 m}+\frac{m w_{c}^{2}}{2} \vec{r}^{2}(j)\right], \\
& U_{N, s} \quad\left(\frac{\Omega}{m w_{c} \eta^{2}(t)}\right)^{\frac{N D}{4}} \prod_{j=1}^{N} \exp \left[\frac{i}{2 \hbar} \frac{m \dot{\eta}(t)}{\eta(t)} \vec{r}^{2}(j)\right] \\
& \quad \times \exp \left[-\frac{1}{2}\left(\ln \frac{m w_{c} \eta^{2}(t)}{\Omega}\right) \vec{r}(j) \cdot \vec{\nabla}(j)\right],
\end{aligned}
$$

one may find the relation

$$
U_{N, s} O_{N, c}\left(\tau(t), w_{c}\right) U_{N, s}^{\dagger}=\left(\frac{d t}{d \tau}\right) O_{N}(t, w(t)) .
$$

If $\chi_{N}(\tau(t))\left[=e^{-i E \tau(t) / \hbar} \chi_{N, 0}(\vec{r}(1), \vec{r}(2), \cdots, \vec{r}(N))\right]$ satisfies the Schrödinger equation

$$
O_{N, c}\left(\tau(t), w_{c}\right) \chi_{N}(\tau(t))=0
$$

a solution of the Schrödinger equation

$$
O_{N}(t, w(t)) \Psi_{N}(t)=0
$$

is thus given as

$$
\begin{aligned}
& \Psi_{N}(t) \\
& =\left(\frac{\Omega}{m w_{c} \eta^{2}(t)}\right)^{\frac{N D}{4}}\left(\frac{u(t)-i v(t)}{\eta(t)}\right)^{E / \hbar} \\
& \quad \times\left(\prod_{j=1}^{N} \exp \left[\frac{i m \dot{\eta}(t)}{2 \hbar \eta(t)} \vec{r}^{2}(j)\right]\right) \\
& \quad \times \chi_{N, 0}(\overrightarrow{\vec{r}}(1), \overrightarrow{\vec{r}}(2), \cdots, \overrightarrow{\vec{r}}(N)),
\end{aligned}
$$

where $\overrightarrow{\tilde{r}}(j)=\sqrt{\frac{\Omega}{m w_{c}}} \frac{\vec{r}(j)}{\eta(t)}$. As in the number density of the NLSE, for a given time, the probability distribution of $\Psi_{N}(t)$ is found from that of $\chi_{N}(\tau(t))$ by globally rescaling the space coordinate along the radial direction with a multiplication factor.

When $w(t)=w_{c}$ (and (30) is satisfied for the NLSE), the squeeze-type operators in $(21,38)$ transform $(23,40)$ into themselves with a replacement of $\tau$ by $t$ (up to a rescaling of the time), respectively, and thus the unitary relations give invariance. If $\eta(t)$ is denoted as $\eta_{c}(t)$ in this case, $\eta_{c}(t)$ is written without losing generality as

$$
\begin{aligned}
\eta_{c}(t)= & \sqrt{\frac{\Omega}{m w_{c}}} \\
& \times \sqrt{A^{2} \cos ^{2} w_{c}\left(t-t_{0}\right)+A^{-2} \sin ^{2} w_{c}\left(t-t_{0}\right)},
\end{aligned}
$$

with a constant $t_{0}$ and a non-zero constant $A . \eta_{c}(t)$ is a periodic function of time with frequency $2 \nu_{c}$, and thus the probability distribution of the wave function obtained from a stationary state of the $N$-body system through the STT has the breathing motion of frequency $2 \nu_{c}$.

The invariance under the STT is possible only when the atoms interact each other through the potential satisfying (27). Such potentials are the inverse-squaretype [17] and the two-dimensional Dirac delta potentials, including other variants from the one-dimensional Dirac delta potential. Since Dirac delta potential cannot be used for the interaction of fermions due to the exclusion principle, the Fermi system with the collective $2 \nu_{c}$ breathing mode should be interpreted as that of no interaction between the atoms (except for a possible long-range interaction through the inversesquare-type potential). In the Tonks-Girardeau limit of the Tonks gas, interacting bosons behaves like noninteracting quasifermions, and the mean-field description has been given by Kolomeisky et al. [18]. Since noninteracting particle systems in one dimension have the invariance, the mean-field equation should also be invariant under the STT, and the equation of Kolomeisky et al. satisfies the requirement of (30).

In order to realize the large breathing mode from a stationary state in a time-independent trap of frequency $\nu_{c}$, one may modulate $w(t)$ until $\eta(t)$ determined by $(27)$ has a large oscillating behavior. In this case a convenient choice for the arbitrary constant $\Omega$ is $\Omega=m w_{c}$, and the limiting case of $A \rightarrow 0$ or $A \rightarrow \infty$ of (43) shows the existences of such modulations. The modulation of frequency has already been widely used in experiments (see, e.g., [1] ). If the amplitude of the center-of-mass oscillation decays in an experiment of a single-component gas using a time-independent harmonic potential, it indicates that the gas is not completely isolated. While a $2 \nu_{c}$ breathing mode is found and a sinusoidal curve is used to fit the size of the breathing gas in Ref. [1], $(35,43)$ imply that the difference between the radius of the breathing motion from the invariance and the sinusoidal curve will be clear for $A>>1$ (or $A<<1$ ) around the time the gases are most compressed. 
In summary, isolated single-component quantum gas in a harmonic potential is considered. The invariance under the DTT shows that the center of mass of the gas moves along a classical trajectory of a harmonic oscillator. For a time-independent potential, it is shown that the harmonic motion of the center has, in fact, been implied by Kohn's theorem [8, 16]. For a Fermi system, through the invariance under the STT, it is shown that appearance of the large radial $2 \nu_{c}$ breathing mode indicates that the atoms do not interact with each other (except for a possible long-range interaction). While some NLSEs and noninteracting Fermi gas in any dimension are invariant under the STT, the collective breathing motion of the one-dimensional gas can be understood from the evolution of an eigenstate of the harmonic oscillator into a coherent state when modulation is applied to the frequency. Alternatively, in one dimension, the collective breathing and oscillating motions in a time-independent potential can be interpreted as the result of using generalized coherent states [12, 13, 14] of a harmonic oscillator in stacking fermions.

\section{Acknowledgments}

The author thanks referees for informing him on Kohn's theorem.
[1] S. Inouye, M. R. Andrews, J. Stenger, H.-J. Miesner, D. M. Stamper-Kurn, and W. Ketterle, Nature 392, 151 (1998).

[2] E.J. Mueller, Phys. Rev. Lett. 93, 190404 (2004); M.D. Girardeau, E.M. Wright, and J.M. Triscari, Phys. Rev. A 63, 033601 (2001).

[3] E. Schrödinger, Naturwissenschaften 14, 664 (1926); K. Husimi, Prog. Theor. Phys. 9, 381 (1953).

[4] D.-Y. Song, Phys. Rev. A 62, 014103 (2000); F.-L. Li, S.J. Wang, A. Weiguny, and D.L Lin, J. Phys. A 27, 985 (1994); A.N. Seleznyova, Phys. Rev. A 51, 950 (1995).

[5] V.M. Perez-Garcia, H. Michinel, J. I. Cirac, M. Lewenstein, and P. Zoller, Phys. Rev. Lett. 77, 5320 (1996).

[6] S.D. Gensemer and D.S. Jin, Phys. Rev. Lett. 87, 173201 (2001); D.S. Jin, J. R. Ensher, M. R. Matthews, C. E. Wieman, and E. A. Cornell, Phys. Rev. Lett. 77, 420 (1996).

[7] S. Stringari, Phys. Rev. Lett. 77, 2360 (1996); M. Edwards, P. A. Ruprecht, K. Burnett, R. J. Dodd, and C. W. Clark, Phys. Rev. Lett. 77, 1671 (1996); C. Menotti and S. Stringari, Phys. Rev. A 66, 043610 (2002).

[8] W. Kohn, Phys. Rev. 123, 1242 (1961); J.F. Dobson, Phys. Rev. Lett. 73, 2244 (1994).

[9] Y. Kagan, E.L. Surkov, and G.V. Shlyapnikov, Phys. Rev. A 54, R1753 (1996); Y.E. Kim and A.L. Zubarev, Phys. Rev. A 67, 015602 (2003).
[10] T.K. Ghosh, Phys. Lett. A 285, 222 (2001); L.P. Pitaevskii and A. Rosch, Phys. Rev. A 55, R853 (1997).

[11] H. Moritz, T. Stöferle, M. Köhl, and T. Esslinger, Phys. Rev. Lett. 91, 250402 (2003).

[12] J.R. Klauder and B.-S. Skagerstam, Coherent States: Applications in Physics and Mathematical Physics (World Scientific, Singapore, 1985); M.M. Nieto, Phys. Lett. A 229, 135 (1997).

[13] D.-Y. Song, Phys. Rev. A 59, 2616 (1999); Phys. Rev. Lett. 85, 1141 (2000).

[14] D.-Y. Song, Phys. Rev. A 63, 032104 (2001); B. Sutherland, Phys. Rev. Lett. 80, 3678 (1998).

[15] S.A. Morgan, R.J. Ballagh, and K. Burnett, Phys. Rev. A 55, 4338 (1997); S.M. Roy and V. Singh, Phys. Rev. D 25, 3413 (1982); Y. Nogami and F.M. Toyama, Phys. Rev. E 49, 4497 (1994).

[16] L. Brey, N.F. Johnson, and B.I. Halperin, Phys. Rev. B 40, 10647 (1989); A.L. Fetter and D. Rokhsar, Phys. Rev. A 57, 1191 (1998).

[17] P.J. Gambardella, J. Math. Phys. 16, 1172 (1975).

[18] E.B. Kolomeisky, T.J. Newman, J.P. Straley, and X. Qi, Phys. Rev. Lett. 85, 1146 (2000); ibid. 86, 4709 (2001). See, also, M.D. Girardeau and E.M. Wright, Phys. Rev. Lett. 84, 5239 (2000). 